\title{
The pan-HDAC inhibitor panobinostat acts as a sensitizer for erlotinib activity in EGFR- mutated and -wildtype non-small cell lung cancer cells
}

Gabriele Greve ${ }^{1,2+}$, Insa Schiffmann ${ }^{1,3+}$, Dietmar Pfeifer ${ }^{1}$, Milena Pantic ${ }^{1}$, Julia Schüler ${ }^{4}$ and Michael Lübbert ${ }^{1,5^{*}}$

\begin{abstract}
Background: The receptor tyrosine kinase (RTK) EGFR is overexpressed and mutated in NSCLC. These mutations can be targeted by RTK inhibitors (TKIs) such as erlotinib. Chromatin-modifying agents may offer a novel therapeutic approach by sensitizing tumor cells to TKls.

Methods: The NSCLC cell lines HCC827 (EGFR mutant, adenocarcinoma), A549 (EGFR wt, adenocarcinoma) and $\mathrm{NCl}-\mathrm{H} 460$ (EGFR wt, large cell carcinoma) were analyzed by SNP6.0 array. Changes in proliferation after panobinostat (LBH-589, PS) and erlotinib treatment were quantified by WST-1 assay and apoptosis by Annexin V/7-AAD flow cytometry. Abundance of target proteins and histone marks (acH3, H3K4me1/2/3) was determined by immunoblotting.

Results: As expected, the EGFR wt cell lines A549 and NCl-H460 were quite insensitive to the growth-inhibitory effect of erlotinib $\left(\mathrm{IC}_{50} 70-100 \mu \mathrm{M}\right)$, compared to $\mathrm{HCC} 827\left(\mathrm{IC}_{50}<0.02 \mu \mathrm{M}\right)$. All three cell lines were sensitive to PS treatment ( $\mathrm{I}_{50}$ : HCC827 $10 \mathrm{nM}, \mathrm{A} 54920 \mathrm{nM}$ and NCl-H460 $35 \mathrm{nM}$ ). The combination of both drugs further reduced proliferation in HCC827 and in A549, but not in NCI-H460. PS alone induced differentiation and expression of p21 WAF1/CIP1 and p53 and decreased CHK1 in all three cell lines, with almost no further effect when combined with erlotinib. In contrast, combination treatment additively decreased pEGFR, pERK and pAKT in A549. Both drugs synergistically induced acH3 in the adenocarcinoma lines. Surprisingly, we also observed induction of H3K4 methylation marks after erlotinib treatment in HCC827 and in A549 that was further enhanced by combination with PS.

Conclusion: PS sensitized lung adenocarcinoma cells to the antiproliferative effects of erlotinib. In these cell lines, the drug combination also had a robust, not previously described effect on histone $\mathrm{H} 3$ acetylation and H3K4 methylation.
\end{abstract}

Keywords: Epigenetic therapy, Non-small cell lung cancer, Erlotinib, Chromatin modification, Histone acetylation, Histone methylation, TKI

\section{Background}

Lung cancer remains the leading cause of cancer-related death worldwide [1]. For stage IIIB/IV non-small cell lung cancer (NSCLC) patients, the median survival with standard chemotherapy is approximately 10 months [2]. While treatment with tyrosine kinase inhibitors (TKIs)

\footnotetext{
* Correspondence: michael.luebbert@uniklinik-freiburg.de

${ }^{\dagger}$ Equal contributors

${ }^{1}$ University of Freiburg Medical Center, Freiburg, Germany

${ }^{5}$ DKTK, German Consortium for Translational Cancer Research, Freiburg, Germany

Full list of author information is available at the end of the article
}

can dramatically prolong survival in a subgroup of patients, their therapeutic index depends heavily on the patients' Epidermal Growth Factor Receptor (EGFR) mutational status [3]. While reversible first-generation TKIs like erlotinib and gefitinib are superior to chemotherapy in EGFR-mutated patients, they show less activity in patients with wildtype (wt) EGFR [4,5]. Different attempts have been undertaken to sensitize EGFR wt NSCLC cells to the antineoplastic effects of TKIs, including combination therapy with epigenetically active drugs [6].

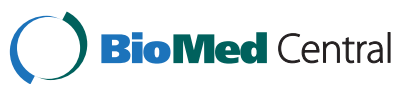

(c) 2015 Greve et al. Open Access This article is distributed under the terms of the Creative Commons Attribution 4.0 International License (http://creativecommons.org/licenses/by/4.0/), which permits unrestricted use, distribution, and reproduction in any medium, provided you give appropriate credit to the original author(s) and the source, provide a link to the Creative Commons license, and indicate if changes were made. The Creative Commons Public Domain Dedication waiver (http://creativecommons.org/publicdomain/zero/1.0/) applies to the data made available in this article, unless otherwise stated. 
Histone deacetylase inhibitors (HDACis) exert their anticancer effects by increasing acetylation of core histones as well as non-histone proteins, thereby influencing gene transcription and ultimately leading to the induction of apoptosis, differentiation or degradation of misfolded proteins [7]. To date, three HDACis, vorinostat (SAHA), romidepsin and panobinostat (LBH-589, PS), have been FDA-approved for the treatment of cutaneous and peripheral T-cell lymphoma and multiple myeloma. In contrast, in solid tumors single-agent treatment with HDACis exhibits only a limited clinical benefit [8]. However, the combination with different cancer therapeutics (chemo-, radio- or antihormonal therapy as well as TKIs), has demonstrated increased efficacy in several settings $[9,10]$.

HDACis and TKIs affect common downstream pathways: both alter the expression of cell cycle regulators such as p21 ${ }^{\text {WAF1/CIP1 }}$, p53 or CHK1 $[11,12]$. Downregulation of $\mathrm{CHK} 1$ was recently shown to be a potential pharmacodynamic biomarker for HDACi response in NSCLC patients and was negatively correlated with the expression of E-cadherin [13]. On the other hand, ECadherin is also an important protein of interest, as its loss leads to both metastatic spread and resistance to TKI treatment, and it was already shown to be upregulated by HDACi in vitro [14]. Thus, high E-Cadherin expression is not only a prognostic marker for a better outcome in lung cancer but also correlates with response to TKIs [14]. Non-toxic treatment regimens combining HDACis and TKIs have been established [13, 15], and first clinical trials showed that the combination therapy is especially effective in patients expressing high ECadherin levels [16].

Recent publications could also demonstrate a crosstalk between HDACi induced histone acetylation and histone methylation that resulted in differentiation and induction of tumor suppressor genes, such as p21 WAF1/CIP1 $[17,18]$.

Here, we utilized three NSCLC cell lines with different genotypes (regarding EGFR and KRAS mutational status, different copy number gains and losses of genes relevant for lung cancer) to investigate the molecular mechanisms of the combination of the pan-HDACi PS with erlotinib. In this study, we could show that the combination of erlotinib with PS indeed exerts additive antineoplastic effects upon the EGFR-mutated adenocarcinoma cell line HCC827 and the EGFR wt adenocarcinoma cell line A549, whereas this effect was not seen in the large-cell carcinoma cell line NCI-H460. Interestingly, the deposition of activating histone marks mediated by HDACi treatment with PS, including histone methylation marks, was enhanced by erlotinib, an effect of the TKI that has not been described previously.

\section{Methods}

\section{Cell lines}

Three non-small cell lung cancer lines HCC827, A549 and NCI-H460 (ATCC, American Type culture collection, Manassas, VA; USA; DSMZ, Braunschweig, Germany) with different histological properties as well as EGFR and KRAS mutational status were cultured in RMPI 1640 (Gibco, Thermo Fisher Scientific Inc.) incl. 10 \% FCS (Biochrome, Merck Millipore, Berlin, Germany) and 100U/ml penicillin/streptomycin (Gibco) at $37{ }^{\circ} \mathrm{C}$ and $5 \% \mathrm{CO}_{2}$.

$\mathrm{HCC} 827$ is an adenocarcinoma line with the E746 A750 EGFR deletion [19]. A549 is of the same histological NSCLC subtype and harbors a KRAS mutation, but is EGFR wildtype [20]. NCI-H460 is a large-cell carcinoma line, also with mutated KRAS and wildtype EGFR [21]. As published in the COSMIC database, none of the three cell line harbors a TP53 mutation (http:// cancer.sanger.ac.uk/ cancergenome/projects/cosmic/).

Since we only used commercially available cell lines, no approval of an appropriate ethics committee was needed.

\section{Treatment of cells, growth inhibition and measurement of apoptosis}

$\mathrm{IC}_{50}$ concentrations of panobinostat (LBH-589, PS, LC laboratories, Woburn, MA, USA) and erlotinib (LC laboratories) were determined by WST-1 assay (Roche Applied Science, Mannheim, Germany). Briefly, $5 \times 10^{2}$ / $\mathrm{ml}$ cells were seeded in 96-well plates, treated on three consecutive days with different concentrations of PS or erlotinib and changes in proliferation were measured according to manufacturer's protocol. $\mathrm{IC}_{50}$ values were reached when cell growth was inhibited to $50 \%$ of the DMSO control. As previously described, the $\mathrm{IC}_{50}$ concentrations for PS were 10nM for HCC827, $20 \mathrm{nM}$ for A549 and $35 \mathrm{nM}$ for NCI-H460 [22]. Since the EGFR wt cells were highly insensitive to erlotinib a dose of $2 \mu \mathrm{M}$ was used. For treatment of EGFR mutated HCC827 cells a non-toxic concentration of $10 \mathrm{nM}$ erlotinib was used.

Both PS and erlotinib were dissolved in DMSO (Sigma) in stock solution of $1 \mathrm{mM}$ and $10 \mathrm{mM}$, respectively, and diluted to the corresponding concentrations directly prior to the treatment for each timepoint.

Cell death was observed by trypan blue exclusion assay and by flow cytometry measurement with Annexin $\mathrm{V}$ (eBioscience, Frankfurt a.M., Germany) and 7-AAD (BD Pharmingen, Heidelberg, Germany) staining.

\section{Western blot}

After treatment with the corresponding $\mathrm{IC}_{50}$-dose of PS and $2 \mu \mathrm{M}$ (10 $\mathrm{nM}$, respectively) erlotinib as single agents and in combination, $5 \times 10^{6}$ NSCLC cells per condition were harvested after 24, 48 and $72 \mathrm{~h}$. DMSO as well as 
medium-only served as negative controls. Whole cell lysis and protein isolation was performed according to protocol (Active Motif) and protein concentration was measured by BCA assay (Thermo Scientific). Equal amounts of protein were separated using 4-12\% gradient Bis/Tris acrylamide gels in the NuPAGE electrophoresis and blotting system (Invitrogen, Thermo Scientific).

Antibodies against phospho- and total ERK, phosphoand total AKT, phospho-and total CHK1, $\beta$-catenin (Cell Signaling Technology), E-cadherin (BD Transduction Laboratories), acetylated histone H3, H3K4me1,-2,-3 (Merck Millipore), p21 ${ }^{\text {WAF1/CIP1 }}$, p53, GAPDH, alpha-tubulin, and HRP-labeled secondary antibodies against goat, rabbit and mouse (Santa Cruz Biotechnology) were used. ECL Plus Western Blotting Detection System (Thermo Scientific) served as substrate for chemoluminescence.

\section{SNP-array analysis}

DNA was isolated from $5 \times 10^{6}$ cells per cell line according to manual with the Blood \& Tissue DNeasy Mini Kit (Qiagen). DNA concentration was measured with NanoDrop1000 (Thermo Scientific).

500 ng of genomic DNA were used for SNP array analysis (Genome-Wide Human SNP Array 6.0 Affymetrix). Copy number variations were analyzed with the Genotyping Console Software (Affymetrix).

[GEO:GSE63784] (http://www.ncbi.nlm.nih.gov/geo/ query/acc.cgi?acc=GSE63784).

\section{Statistics}

Statistical analysis of data was performed using the GraphPad Prism 5.0 software with Student's $t$-test to test differences between two treatment groups and a linear regression model for the identification of the corresponding $\mathrm{IC}_{50}$ values.

\section{Results}

Characterization of three NSCLC cell lines reveals copy number variations in oncogenes and tumor suppressor genes

Before treatment of the cell lines HCC827, A549 and NCI-H460, we wished to confirm and extend the genetic information given by the provider. We analyzed copy number $(\mathrm{CN})$ variations by SNP array, since copy number amplifications of EGFR, MYC and KRAS and TP63 are often described in NSCLC. Specifically EGFR mutated cells like HCC827 display an amplification of the oncogene [23] which we could confirm (Table 1). Similarly, amplification of $M Y C$ was validated for the NCI$\mathrm{H} 460$ cells (with a concomitant $\mathrm{CN}$ reduction of EGFR). Amplifications of KRAS could be confirmed for A549 (KRAS mut) and the KRAS wt HCC287 [24]. None of the three cell lines showed amplifications of $M E T$, which are closely linked to TKI resistance $[25,26]$. No CN gains or losses were detected for $A L K, B R A F$ and $P I 3 K$, (Table 1).

\section{Panobinostat enhances the antiproliferative effect of erlotinib in EGFR-mutant and -wildtype cell lines}

We next examined growth-inhibitory effects of erlotinib and PS, alone and in combination. NSCLC patients usually receive a daily dose of $150 \mathrm{mg}$ erlotinib, which results in plasma levels between 1 and $3 \mu \mathrm{M}$ [27]. Thus we chose to treat the EGFR wildtype cells with an erlotinib concentration of $2 \mu \mathrm{M}$. Additionally, we performed extensive dose-findings to determine the most effective but also least toxic doses for PS in combination with erlotinib (Fig. 1a).

As expected, proliferation of A549 and $\mathrm{NCl}-\mathrm{H} 460$ cells was not inhibited by erlotinib alone, while EGFR mutant HCC827 cells showed striking growth inhibition already at $10 \mathrm{nM}$ (Fig. 1b). Treatment with PS at nanomolar doses impaired proliferation in all three cell lines, with HCC827 being most sensitive [22]. When combining PS and erlotinib, a time- and dose-dependent growth inhibition could be observed, with a significantly additive effect of PS across the entire dose range in HCC827 and A549. In NCI-H460, erlotinib showed no additional effect. (Fig. 1b). Inhibition of proliferation was not accompanied by an increase in cytotoxicity and apoptosis (Fig. 2).

\section{Combined panobinostat/erlotinib treatment reduces phospho-EGFR}

Binding of its ligands leads to EGFR dimerization and autophosphorylation, allowing the recruitment of its downstream targets. Therefore, phosphorylation is an indicator for activated EGFR.

As shown in Fig. 3a, after $72 \mathrm{~h}$ PS slightly reduced phospho-EGFR in both EGFR wt cell lines whereas no

Table 1 Copy number variations of oncogenes and tumor suppressors in three NSCLC cell lines

\begin{tabular}{|c|c|c|c|c|c|c|c|c|c|c|}
\hline gene & ALK & BRAF & CDKN1A & EGFR & KRAS & MET & MYC & TP53 & TP63 & $\mathrm{Pl} 3 \mathrm{~K}$ \\
\hline HCC827 & $n$ & $n$ & $n$ & 4 & 3 & $n$ & 4 & 3 & $n$ & $n$ \\
\hline A549 & $n$ & $n$ & 1 & 3 & 3 & $n$ & 3 & $n$ & 1 & $\mathrm{n}$ \\
\hline $\mathrm{NCl}-\mathrm{H} 460$ & $\mathrm{n}$ & $\mathrm{n}$ & 1 & 1 & $\mathrm{n}$ & $\mathrm{n}$ & 4 & $\mathrm{n}$ & $\mathrm{n}$ & $\mathrm{n}$ \\
\hline
\end{tabular}

Copy numbers of selected genes in $\mathrm{HCC} 827, \mathrm{~A} 549$ and $\mathrm{NCl}-\mathrm{H} 460$ were determined by Affymetrix SNP Array 6.0

$0=$ complete loss, $1=50 \%$ loss, $n=$ no loss or gain, $3=50 \%$ gain and $4=100 \%$ gain of genomic material, relative to ploidy (all percentages are approximate values computed with Affymetrix Genotyping Console Software) 

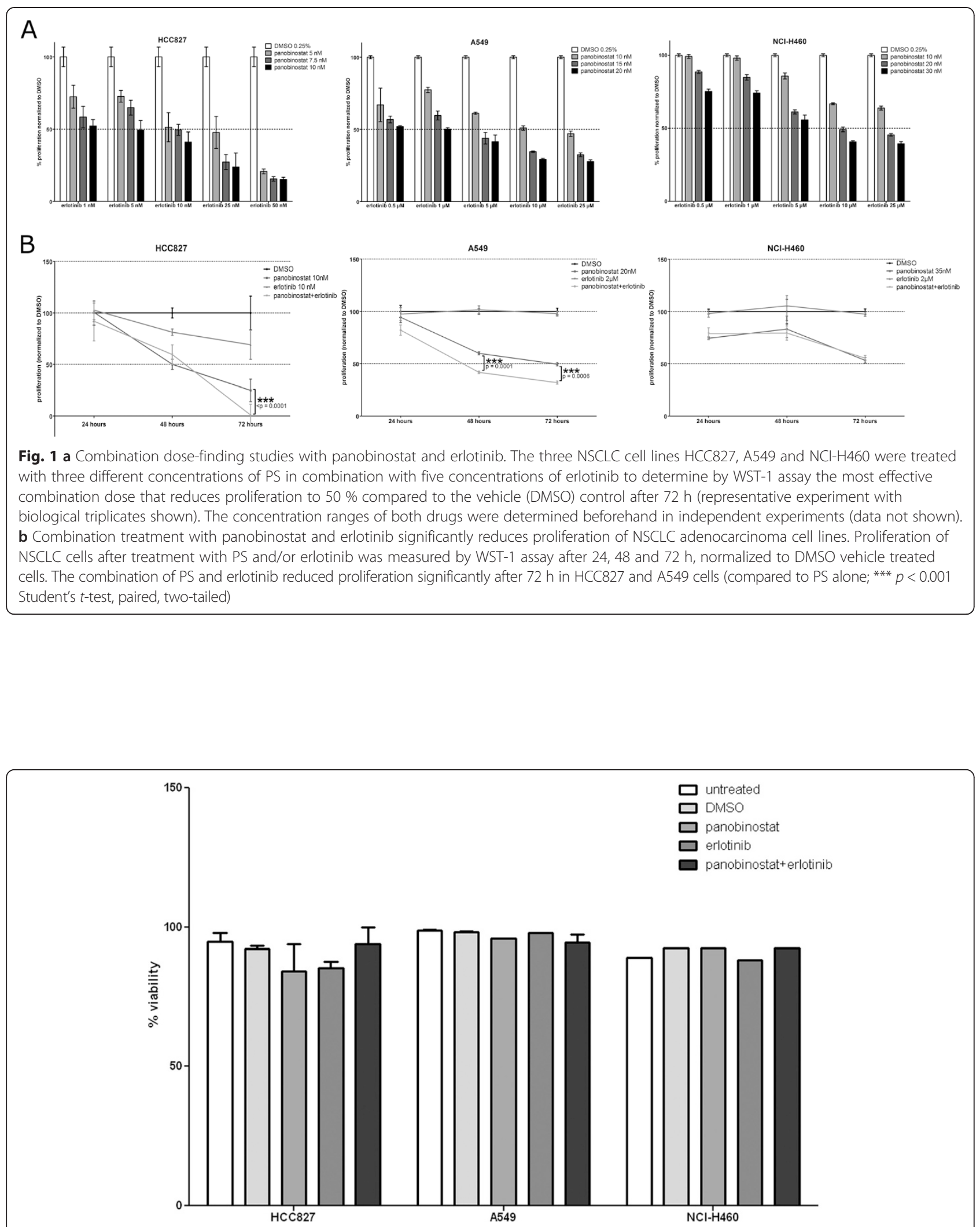

Fig. 2 Combination treatment with panobinostat and erlotinib shows no increase in cytotoxicity after $72 \mathrm{~h}$. Viability of NSCLC cells after $72 \mathrm{~h}$ of treatment with PS and/or erlotinib measured via Trypan blue exclusion assay. No significant induction of cell death was measured. This was also validated by flow cytometry with Annexin $V$ and 7-AAD (data not shown) 


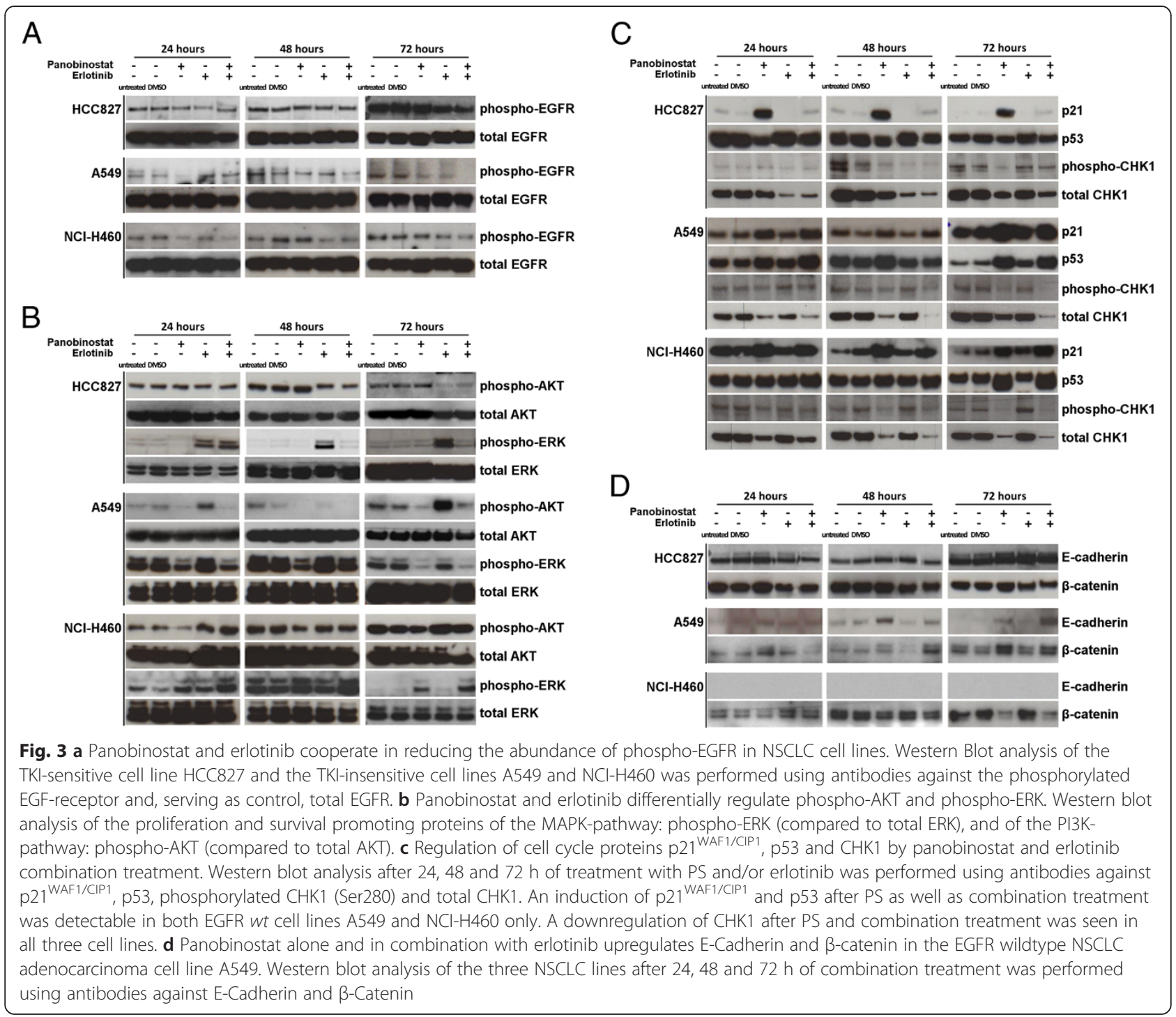

effect could be detected in HCC827. As expected, a response to erlotinib could be seen in HCC827, but also A549 showed a decrease in phospho-EGFR. Combining the two compounds further downregulated phosphoEGFR in HCC827, A549 and, to a lesser extent, also in NCI-H460.

\section{Panobinostat and erlotinib affect the expression of downstream targets of the EGFR pathway}

EGFR is located upstream of a signaling cascade that includes the PI3K/AKT- and RAS/MAPK-pathways and regulates cell differentiation and proliferation. Western Blot was performed to investigate the effects of erlotinib \pm PS on the expression and phosphorylation of EGFR downstream proteins AKT and ERK.

In HCC827, erlotinib diminished phospho-AKT almost completely; this effect could not be further increased by PS. Unexpectedly, single agent erlotinib was not only unable to decrease the already low, phospho-ERK levels, but rather induced phosphorylation of ERK. In A549, erlotinib had no effect on phospho-ERK but induced the expression of phospho-AKT, whereas PS reduced phosphorylation of AKT and ERK. In NCI-H460, erlotinib had no effect on phospho-AKT or phospho-ERK. PS alone could only slightly reduce the phosphorylation of AKT, but, in contrast, increased phosphorylation of ERK after $72 \mathrm{~h}$. As in A549, no cooperative effect of the combination could be detected (Fig. 3b).

In EGFR mutated cells, erlotinib counteracts panobinostatinduced increase of p2 $1^{\text {WAF1/CIP1 }}$ and p53 and decrease of CHK1

The p53 pathway reacts to different stress signals caused by e.g. DNA damage or hypoxia. Its downstream targets include the CDK inhibitor $\mathrm{p} 21^{\mathrm{WAF} 1 / \mathrm{CIP} 1}$, which however can also be induced p53-independently [28]. Both 
cooperatively downregulate CHK1 [29]. Additionally, AKT further inhibits CHK1 function by phosphorylation of Ser280. As already shown, HDAC inhibition leads to an upregulation of $\mathrm{p} 21^{\mathrm{WAF} 1 / \mathrm{CIP} 1}$, acetylation and induction of p53 and reduction of CHK1 [12, 30].

In all three cell lines, PS led to a strong $\mathrm{p} 21^{\mathrm{WAF} 1 / \mathrm{CIP} 1}$ induction. Whereas in NCI-H460 p21 WAF1/CIP1 was increased even further by PS + erlotinib, in HCC827 the combination antagonized this induction almost entirely. In A549 combination treatment had almost no additional effect (Fig. 3c). In the HCC827 cell line, erlotinib led to a slight increase of p53 after $72 \mathrm{~h}$, whereas with PS or the combination of both drugs p53 levels were modestly decreased. In A549 and NCI-H460, a marked increase of p53 expression was only apparent after $72 \mathrm{~h}$ of PS and combination treatment (no additive effect) (Fig. 3c, middle and lower panel).

CHK1 decreased markedly in all three cell lines after $72 \mathrm{~h}$ of HDACi treatment. In HCC827 and A549 add-on of erlotinib further enhanced this effect. In line with the AKT immunoblot results, phosphoCHK1 was slightly increased in all three cell lines after erlotinib treatment.

\section{The panobinostat and erlotinib combination induces an epithelial phenotype in $\mathrm{HCC} 827$ and A549 cells}

Epithelial to mesenchymal transition (EMT) plays a major role in the metastatic behavior of NSCLC. Also, loss of the epithelial marker E-cadherin is a hallmark in the development of TKI resistance [14]. In HCC827, PS modestly upregulated E-cadherin, indicative of some EMT induction (Fig. 3d, upper panel), as did erlotinib and the combination treatment $(72 \mathrm{~h})$. PS alone modestly induced $\beta$-catenin expression, whereas the combination with erlotinib had the opposite effect (which appeared to outweigh the effect of HDAC inhibition). A549 did not disclose E-cadherin or $\beta$-catenin induction by erlotinib, whereas PS, both alone and in combination with erlotinib, led to an increased expression of both proteins. NCI-H460 showed a different response: PS alone and in combination with erlotinib reduced $\beta$ catenin. (No E-cadherin expression was detectable in this cell line, neither before nor after treatment, Fig. 3d, lower panel) [22, 31].

\section{Erlotinib enhances panobinostat-induced acetylation of histone $\mathrm{H} 3$}

As expected, PS robustly induced acetyl-histone H3 (acH3). Erlotinib had no effect on H3 acetylation, but it enhanced the effect of PS on acH3 (in HCC827 and A549). In NCI-H460, it modestly induced acH3 after $24 \mathrm{~h}$, but the combination treatment did not lead to an additive effect compared to PS alone (Fig. 4).
The combination of panobinostat and erlotinib leads to synergistic induction of activating histone methylation marks in HCC827 and A549

Others [32] as well as ourselves [33], could show that HDAC inhibitors also exert an effect on different histone methylation marks. Therefore we also investigated the histone marks mono-, di-, and trimethylated lysine 4 of histone $\mathrm{H} 3$ (H3K4me1/2/3, respectively) by Western blot (Fig. 4). Baseline H3K4 methylation was hardly detectable in all three cell lines. PS induced H3K4me1/2/3 in all three cell lines. Surprisingly, also erlotinib had a positive effect upon methylation of this residue, particularly in HCC827 and, to a lesser degree, in A549 cells. Strikingly, the drug combination exerted a robust synergistic effect on the expression of all three methylation steps of $\mathrm{H} 3 \mathrm{~K} 4$ in both adenocarcinoma cell lines (Fig. 4).

To investigate if there was a genomic basis for this effect, we also checked for copy number alterations in genes that play important roles in histone methylation by removing or adding methyl groups to H3K4 (i.e. histone demethylases and methyltransferases). We found gains for KDM4A and KDM5A (HCC827) and of $K D M 5 B$ (NCI-H460). Losses were detected of $K D M 1 A$ and KDM4A (A549) and KDM1A and $K D M 2 A$ (NCI-H460). No copy number changes could be seen for histone methyltransferases SETD1A, $S E T D 1 B, K M T 2 A / B / C / D$ (see Table 2). As we compared these data to already published expression data [34], we could not find a direct correlation between copy numbers and mRNA expression (Fig. 5). These findings suggest that differential methylation of H3K4 is not regulated by copy number alterations of the particular demethylases and methyltransferases. Both PS and erlotinib, alone and in combination, could robustly induce active histone marks independently of genomic amplifications or losses of these enzymes.

\section{Discussion}

The introduction of tyrosine kinase inhibitors antagonizing overactivated or amplified EGFR has strikingly expanded the therapeutic armamentarium in non-small cell lung cancer. However, development of resistance to TKIs is a major clinical problem, since it occurs in almost all patients receiving these drugs for prolonged periods. Therefore strategies to overcome secondary resistance to TKIs have been investigated. These include the usage of epigenetically active agents such as HDACis. In NSCLC, this structurally diverse group of compounds has been tested pre-clinically and in part also already clinically. While single agent HDACi treatment has only modest activity, drug combinations with chemotherapy have been studied, with some trials being 


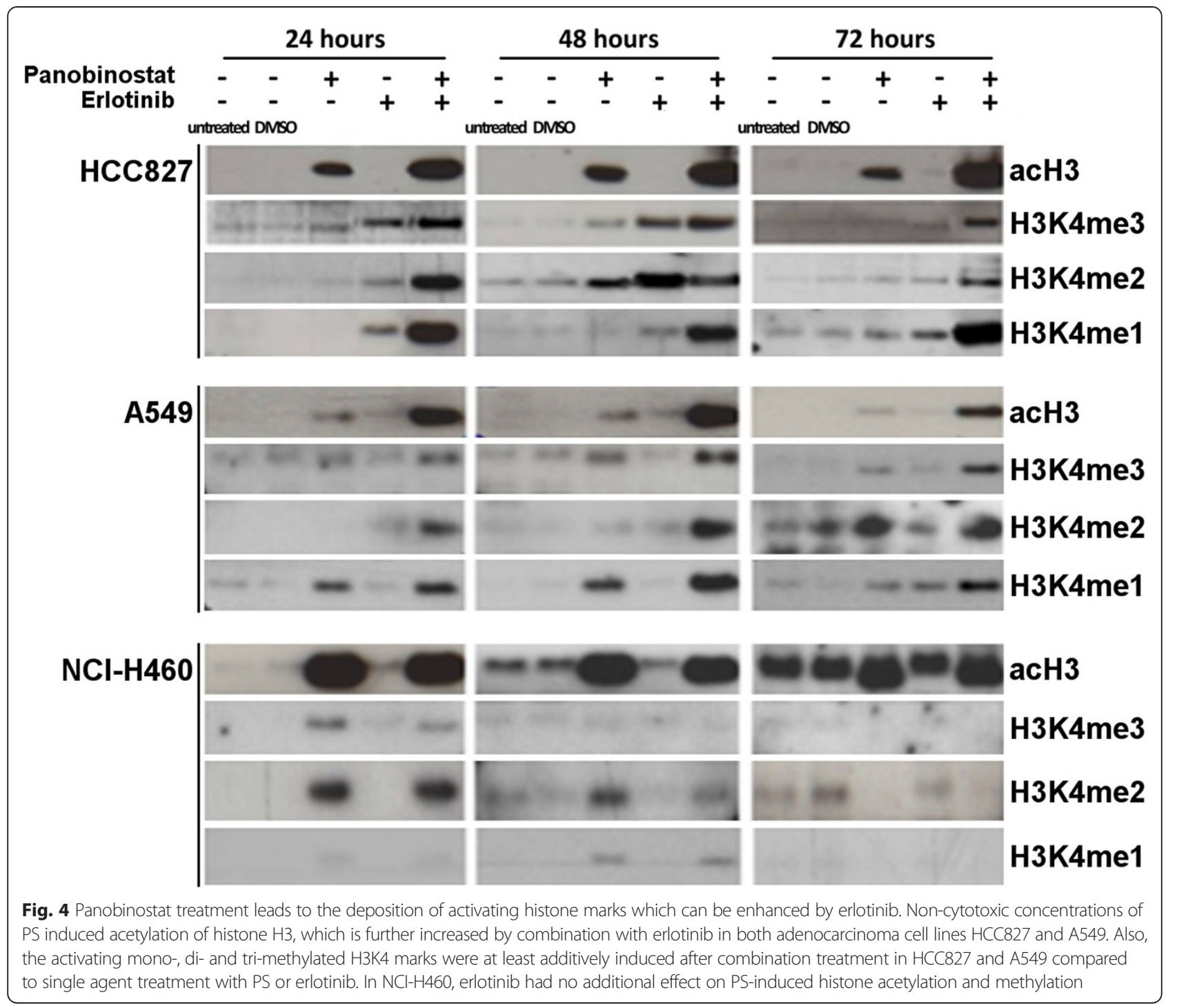

positive [9], others being limited by toxicity of the combination [35]. A single phase II study combining erlotinib with the class 1 selective HDACi entinostat has resulted in prolonged progression-free survival in NSCLC patients harboring high E-cadherin levels, irrespective of the EGFR genotype [16]. The combination of a pan-HDAC inhibitor like PS with erlotinib has so far been investigated in a phase I trial in aerodigestive tract tumors, which suggested that especially TKI-naïve
EGFR-mutated patients might benefit from the combination therapy [13].

In the present study, we attempted to unravel mechanisms of action of the combination treatment of PS with erlotinib in NSCLC cell lines representing different histological subtypes and different genotypes. The combination treatment revealed variable responses: whereas proliferation of the adenocarcinoma cell lines was significantly reduced, the large-cell carcinoma cell line did not respond.

Table 2 Copy numbers of H3K4-specific demethylases and methyltransferases

\begin{tabular}{|c|c|c|c|c|c|c|c|c|c|c|c|c|c|}
\hline gene & KDM1A & KDM2A & KDM4A & KDM5A & KDM5B & KDM5C & KDM5D & SETD1A & SETD1B & KMT2A & KMT2B & KMT2C & KMT2D \\
\hline HCC827 & $n$ & $n$ & 3 & 3 & $n$ & $n$ & $n$ & $n$ & $n$ & $n$ & $n$ & $n$ & $n$ \\
\hline A549 & 1 & $n$ & 1 & $n$ & $n$ & $n$ & $n$ & $n$ & $n$ & $n$ & $\mathrm{n}$ & $n$ & $n$ \\
\hline $\mathrm{NCl}-\mathrm{H} 460$ & 1 & 1 & $n$ & $n$ & 3 & $\mathrm{n}$ & $\mathrm{n}$ & $n$ & $n$ & $n$ & $n$ & $n$ & $\mathrm{n}$ \\
\hline
\end{tabular}

Evaluation of copy numbers of seven demethylases and six methyltransferases that specifically target lysine 4 residues of histone $\mathrm{H} 3$ (H3K4)

$0=$ complete loss, $1=50 \%$ loss, $n=$ no loss or gain, $3=50 \%$ gain and $4=100 \%$ gain of genomic material, relative to ploidy (all percentages are approximate values computed with Affymetrix Genotyping Console Software) 


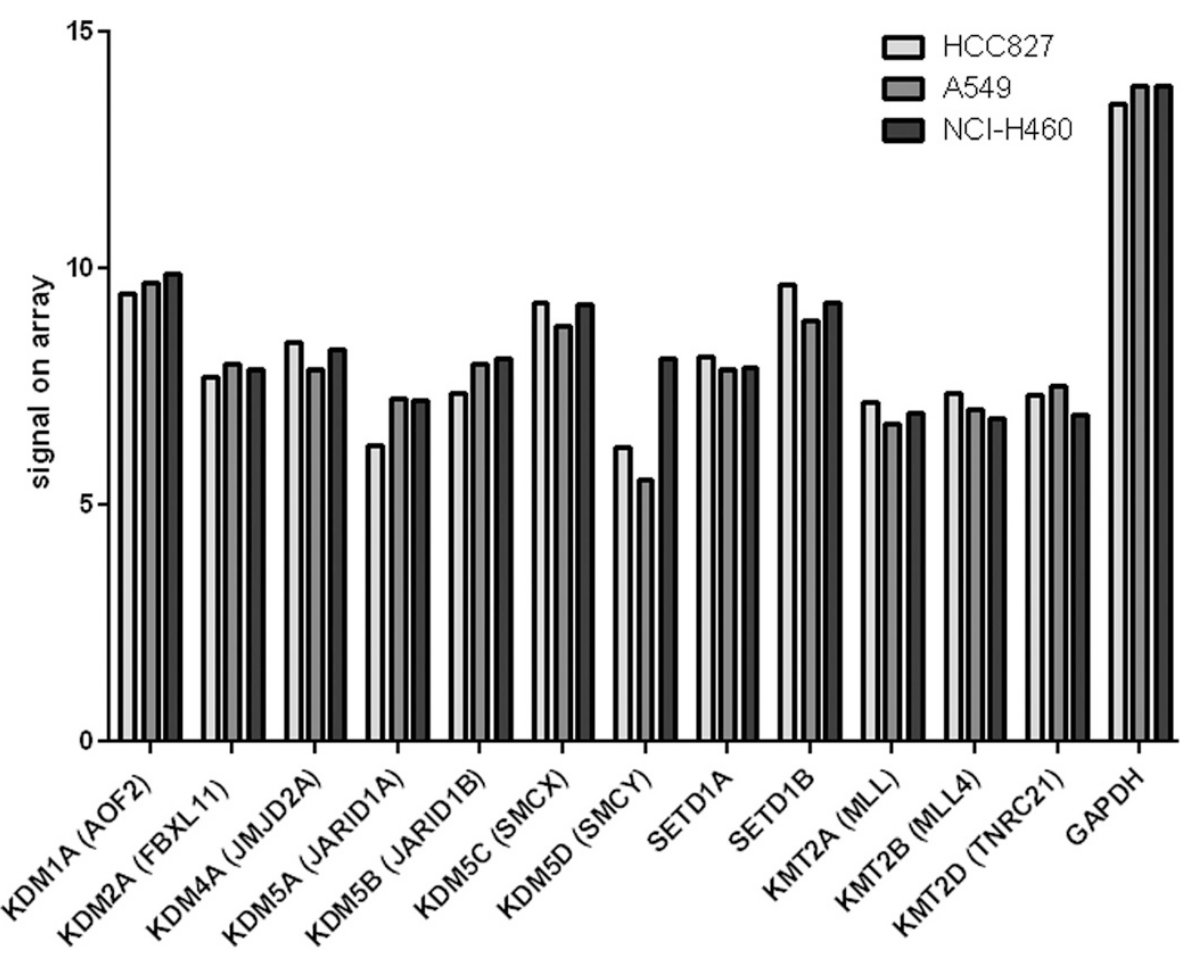

Fig. 5 Expression levels of the epigenetically active enzymes of Table 2. mRNA baseline expression levels of the seven H3K4 demethylases and five methyltransferases already depicted in Table 2. Expression levels of the three cell lines were determined by Affymetrix HG-U133A epression arrays by Coldren et al. 2006 and signal intensities as reposited at the GEO database (GSE4342) were plotted. [34]

We also hypothesized that the inhibitory effect of erlotinib on EGFR phosphorylation would be enhanced by the HDAC inhibitor, which could indeed be demonstrated for A549 cells and, to a much lesser degree, for HCC827. Cooperative negative effects on downstream signals, e.g. ERK and AKT were also seen only in these two cell lines. Interestingly, in HCC827 erlotinib hardly reduced phospho-EGFR levels and even upregulated phospho-ERK expression. These findings suggest the, already described, partial outgrowth of a TKI-insensitive cell clone. NCI-H460 displayed no change in phosphoAKT expression and phospho-ERK was even upregulated after PS (alone and in combination with erlotinib) treatment, underlining the insensitivity to TKIs as seen in the proliferation experiments.

Similarly, induction of p $21^{\text {WAF1/CIP1 }}$ and $\mathrm{p} 53$ varied broadly, with EGFR wt cell lines being overall more sensitive. Here, especially NCI-H460 showed an additive effect of both drugs on p21 ${ }^{\text {WAF1/CIP1 }}$ expression and p53 was upregulated by PS only. This previously described [28] PS-induced induction of p $21^{\mathrm{WAF} 1 / \mathrm{CIP} 1}$ and $\mathrm{p} 53$ was also present in A549. In HCC827 cells erlotinib counteracted the impact of PS on p21 ${ }^{\text {WAF1/CIP1 }}$ expression; p53 was reduced by PS with no effect of erlotinib. We could also confirm the correlation between HDAC inhibition and

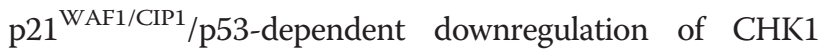
[12]. Additionally, we could also detect a modest increase in (and thus inhibition of) phospho-CHK1 after erlotinib treatment. Taken together, this crosstalk between p21 WAF1/ CIP1/p53 upregulation and CHK1 downregulation and inhibition provides a reasonable mechanism for the observed growth inhibition after HDACi treatment.

HDAC inhibitors have been demonstrated to also affect histone methylation, as we could recently show in a model of acute myeloid leukemia [33]. Therefore we further wished to ask whether the antiproliferative effects of PS were associated with deposition of activating histone lysine methylation marks. Indeed, we could demonstrate that global H3K4 methylation levels, which are usually associated with accessible chromatin, were increased by PS in all three cell lines. Unexpectedly, erlotinib as a single agent also induced histone lysine methylation, in HCC827 and A549. Combining both drugs resulted in a synergistic effect on H3K4 methylation (and H3 acetylation), which strongly mirrored the antiproliferative activity of this combination. To the best of our knowledge, a robust effect of erlotinib upon histone methylation has not been previously described. Possibly, induction of histone methyltransferase expression or inhibition of histone demethylase expression may be responsible for this effect. 


\section{Conclusions}

Pan-HDAC inhibitors such as PS may provide a viable option to (re) sensitize NSCLC cells, particularly of adenocarcinoma subtype with EGFR mutations, to the antiproliferative effects of the TKI erlotinib, warranting further development of this approach within a clinical phase II trial. Our study suggests that the mechanism of action of resensitization involves reactivation of different sets of tumor suppressor genes. We were able to show that this is mediated by inducing an active chromatin configuration via deposition of activating histone marks, such as $\mathrm{H} 3$ acetylation and H3K4 methylation. This general strategy may become even more relevant in the future, since resistance to EGFR T790M-specific third generation kinase inhibitors, like AZD9291 and Rociletinib (CO-1686), already occur in the clinic which needs to be overcome or might even be prevented [36].

\section{Competing interests}

The authors declare that they have no competing interests.

\section{Authors' contributions}

GG designed and performed experiments, analyzed and interpreted data, drafted and revised the manuscript. Is performed experiments, analyzed and interpreted data, drafted and revised the manuscript. DP designed and performed experiments, analyzed data and revised the manuscript. MP designed and performed experiments, analyzed data and revised the manuscript. JS designed and performed experiments and analyzed data. ML designed the study, interpreted data, drafted and revised the manuscript. All authors read and approved the final manuscript.

\section{Acknowledgements}

We thank Tobias Ma and Anna Löhr for technical assistance as well as Gregor Klaus for helpful discussions. We especially want to thank Roland Schüle and Manfred Jung for their continued constructive comments. This work was supported by the German Research Council (DFG, CRC 992 MEDEP, Project C04) and the German Consortium for Translational Cancer Research (DKTK). The article processing charge was funded by the Albert Ludwigs University Freiburg in the funding programme Open Access Publishing.

\section{Author details \\ ${ }^{1}$ University of Freiburg Medical Center, Freiburg, Germany. ${ }^{2}$ University of Freiburg, Faculty of Biology, Freiburg, Germany. ${ }^{3}$ University of Freiburg, Faculty of Medicine, Freiburg, Germany. ${ }^{4}$ Department for in vivo Tumorbiology, Oncotest GmbH, Freiburg, Germany. ${ }^{5}$ DKTK, German Consortium for Translational Cancer Research, Freiburg, Germany.}

Received: 11 December 2014 Accepted: 1 December 2015 Published online: 16 December 2015

\section{References}

1. Siegel R, Ma J, Zou Z, Jemal A. Cancer statistics, 2014. CA Cancer J Clin. 2014:64:9-29.

2. Ries LAG, Reichman ME, Lewis DR, Hankey BF, Edwards BK. Cancer Survival and Incidence from the Surveillance, Epidemiology, and End Results (SEER) Program. Oncologist. 2003:8:541-52.

3. Lynch TJ, Bell DW, Sordella R, Gurubhagavatula S, Okimoto RA, Brannigan BW, et al. Activating Mutations in the Epidermal Growth Factor Receptor Underlying Responsiveness of Non-Small-Cell Lung Cancer to Gefitinib. N Engl J Med. 2004;350:2129-39.

4. Mok TS, Wu Y-L, Thongprasert S, Yang C-H, Chu D-T, Saijo N, et al. Gefitinib or Carboplatin-Paclitaxel in Pulmonary Adenocarcinoma. N Engl J Med. 2009;361:947-57

5. Garassino MC, Martelli O, Broggini M, Farina G, Veronese S, Rulli E, et al. Erlotinib versus docetaxel as second-line treatment of patients with advanced non-small-cell lung cancer and wild-type EGFR tumours (TAILOR): a randomised controlled trial. Lancet Oncol. 2013;14:981-8.

6. Tellez CS, Grimes MJ, Picchi MA, Liu Y, March TH, Reed MD, et al. SGI-110 and entinostat therapy reduces lung tumor burden and reprograms the epigenome. Int J Cancer. 2014;135:2223-31.

7. Keller K, Jung M. Histone Deacetylase (HDAC) Inhibitors in Recent Clinical Trials for Cancer Therapy. In: Lübbert M, Jones PA, editors. Epigenetic Therapy of Cancer. Berlin, Heidelberg: Springer Berlin Heidelberg; 2014. p. 227-55.

8. Kumagai T, Wakimoto N, Yin D, Gery S, Kawamata N, Takai N, et al. Histone deacetylase inhibitor, suberoylanilide hydroxamic acid (Vorinostat, SAHA) profoundly inhibits the growth of human pancreatic cancer cells. Int J Cancer. 2007;121:656-65.

9. Ramalingam SS, Maitland ML, Frankel P, Argiris AE, Koczywas M, Gitlitz B, et al. Carboplatin and Paclitaxel in Combination With Either Vorinostat or Placebo for First-Line Therapy of Advanced Non-Small-Cell Lung Cancer. J Clin Oncol. 2010;28:56-62.

10. Jones DR, Moskaluk CA, Gillenwater HH, Petroni GR, Burks SG, Philips J, et al. Phase I Trial of Induction Histone Deacetylase and Proteasome Inhibition Followed by Surgery in Non-small Cell Lung Cancer. J Thorac Oncol Off Publ Int Assoc Study Lung Cancer. 2012;7:1683-90.

11. Derks S, van Engeland M. Epigenetic Disturbances in Colorectal Cancer. In: Lübbert M, Jones PA, editors. Epigenetic Therapy of Cancer. Berlin, Heidelberg: Springer Berlin Heidelberg; 2014. p. 283-98.

12. Brazelle W, Kreahling JM, Gemmer J, Ma Y, Cress WD, Haura E, et al. Histone Deacetylase Inhibitors Downregulate Checkpoint Kinase 1 Expression to Induce Cell Death in Non-Small Cell Lung Cancer Cells. PLoS One. 2010;5:e14335.

13. Gray JE, Haura E, Chiappori A, Tanvetyanon T, Williams CC, Pinder-Schenck $M$, et al. A Phase I, Pharmacokinetic, and Pharmacodynamic Study of Panobinostat, an HDAC Inhibitor, Combined with Erlotinib in Patients with Advanced Aerodigestive Tract Tumors. Clin Cancer Res. 2014;20:1644-55.

14. Witta SE, Gemmill RM, Hirsch FR, Coldren CD, Hedman K, Ravdel L, et al. Restoring E-Cadherin Expression Increases Sensitivity to Epidermal Growth Factor Receptor Inhibitors in Lung Cancer Cell Lines. Cancer Res. 2006;66:944-50.

15. Gerber DE, Boothman DA, Fattah FJ, Dong Y, Zhu H, Skelton RA, et al. Phase 1 study ofromidepsin plus erlotinib in advanced non-small cell lung cancer. Lung Cancer. 2015. [Epub ahead of print].

16. Witta SE, Jotte RM, Konduri K, Neubauer MA, Spira Al, Ruxer RL, et al. Randomized Phase II Trial of Erlotinib With and Without Entinostat in Patients With Advanced Non-Small-Cell Lung Cancer Who Progressed on Prior Chemotherapy. J Clin Oncol. 2012;30:2248-55.

17. Huang P-H, Plass C, Chen C-S. Effects of Histone Deacetylase Inhibitors on Modulating H3K4 Methylation Marks - A Novel Cross-Talk Mechanism between Histone-Modifying Enzymes. Mol Cell Pharmacol. 2011;3:39-43.

18. Blagitko-Dorfs N, Jiang Y, Duque-Afonso J, Hiller J, Yalcin A, Greve G, et al. Epigenetic Priming of AML Blasts for All-trans Retinoic Acid-Induced Differentiation by the HDAC Class-I Selective Inhibitor Entinostat. PLoS One. 2013;8:e75258.

19. Virmani AK, Fong KM, Kodagoda D, Mclntire D, Hung J, Tonk V, et al. Allelotyping demonstrates common and distinct patterns of chromosomal loss in human lung cancer types. Genes Chromosomes Cancer. 1998;21:308-19.

20. Lieber M, Smith B, Szakal A, Nelson-Rees W, Todaro G. A continuous tumorcell line from a human lung carcinoma with properties of type II alveolar epithelial cells. Int J Cancer. 1976;17:62-70.

21. Gazdar AF, Carney DN, Russell EK, Sims HL, Baylin SB, Bunn PA, et al. Establishment of Continuous, Clonable Cultures of Small-Cell Carcinoma of the Lung Which Have Amine Precursor Uptake and Decarboxylation Cell Properties. Cancer Res. 1980;40:3502-7.

22. Greve G, Schiffmann I, Lübbert M. Epigenetic priming of non-small cell lung cancer cell lines to the antiproliferative and differentiating effects of all-trans retinoic acid. J Cancer Res Clin Oncol. 2015.

23. Sethi G, Ahn KS, Chaturvedi MM, Aggarwal BB. Epidermal growth factor (EGF) activates nuclear factor-kB through IkBa kinase-independent but EGF receptor-kinase dependent tyrosine 42 phosphorylation of IkBa. Oncogene. 2007;26:7324-32

24. Modrek B, Ge L, Pandita A, Lin E, Mohan S, Yue P, et al. Oncogenic Activating Mutations Are Associated with Local Copy Gain. Mol Cancer Res. 2009;7:1244-52. 
25. Kubo T, Yamamoto H, Lockwood WW, Valencia I, Soh J, Peyton M, et al. MET gene amplification or EGFR mutation activate MET in lung cancers untreated with EGFR tyrosine kinase inhibitors. Int J Cancer J Int Cancer. 2009;124:1778-84.

26. Suda K, Murakami I, Katayama T, Tomizawa K, Osada H, Sekido Y, et al. Reciprocal and Complementary Role of MET Amplification and EGFR T790M Mutation in Acquired Resistance to Kinase Inhibitors in Lung Cancer. Clin Cancer Res. 2010;16:5489-98.

27. Motoshima K, Nakamura Y, Sano K, Ikegami Y, Ikeda T, Mizoguchi K, et al. Phase II trial of erlotinib in patients with advanced non-small-cell lung cancer harboring epidermal growth factor receptor mutations: additive analysis of pharmacokinetics. Cancer Chemother Pharmacol. 2013;72:1299-304.

28. George P, Bali P, Annavarapu S, Scuto A, Fiskus W, Guo F, et al. Combination of the histone deacetylase inhibitor LBH589 and the hsp90 inhibitor 17-AAG is highly active against human CML-BC cells and AML cells with activating mutation of FLT-3. Blood. 2005;105:1768-76.

29. Gottifredi V, Karni-Schmidt O, Shieh S-Y, Prives C. p53 Down-Regulates CHK1 through p21 and the Retinoblastoma Protein. Mol Cell Biol. 2001;21:1066-76.

30. Luo J, Su F, Chen D, Shiloh A, Gu W. Deacetylation of p53 modulates its effect on cell growth and apoptosis. Nature. 2000;408:377-81.

31. Ohira T, Gemmill RM, Ferguson K, Kusy S, Roche J, Brambilla E, et al. WNT7a induces E-cadherin in lung cancer cells. Proc Natl Acad Sci U S A. 2003;100:10429-34.

32. Nightingale KP, Gendreizig S, White DA, Bradbury C, Hollfelder F, Turner BM. Cross-talk between Histone Modifications in Response to Histone Deacetylase Inhibitors. J Biol Chem. 2007;282:4408-16.

33. Duque-Afonso J, Yalcin A, Berg T, Abdelkarim M, Heidenreich O, Lübbert M. The HDAC class I-specific inhibitor entinostat (MS-275) effectively relieves epigenetic silencing of the LAT2 gene mediated by AML1/ETO. Oncogene. 2011;30:3062-72

34. Coldren CD, Helfrich BA, Witta SE, Sugita M, Lapadat R, Zeng C, et al. Baseline Gene Expression Predicts Sensitivity to Gefitinib in Non-Small Cell Lung Cancer Cell Lines. Mol Cancer Res. 2006;4:521-8.

35. Schneider BJ, Kalemkerian GP, Bradley D, Smith DC, Egorin MJ, Daignault S, et al. Phase I study of vorinostat (suberoylanilide hydroxamic acid, NSC 701852) in combination with docetaxel in patients with advanced and relapsed solid malignancies. Invest New Drugs. 2012;30:249-57.

36. Politi K, Ayeni D, Lynch T. The Next Wave of EGFR Tyrosine Kinase Inhibitors Enter the Clinic. Cancer Cell. 2015;27:751-3.

\section{Submit your next manuscript to BioMed Central and we will help you at every step:}

- We accept pre-submission inquiries

- Our selector tool helps you to find the most relevant journal

- We provide round the clock customer support

- Convenient online submission

- Thorough peer review

- Inclusion in PubMed and all major indexing services

- Maximum visibility for your research

Submit your manuscript at www.biomedcentral.com/submit
C Biomed Central 